\title{
Anti-correlation between multiplicity and orbital properties in exoplanetary systems as a possible record of their dynamical histories (Corrigendum)
}

\author{
A. Zinzi ${ }^{1,2}$ and D. Turrini ${ }^{3,4}$ \\ ${ }^{1}$ Space Science Data Center (SSDC) - ASI, Via del Politecnico snc, 00133 Roma, Italy \\ e-mail: angelo.zinzi@ssdc.asi.it \\ 2 INAF-OAR, Via Frascati 33, 00078 Monte Porzio Catone, Italy \\ 3 INAF-IAPS, Via del Fosso del Cavaliere 100, 00133 Roma, Italy \\ e-mail: diego.turrini@iaps.inaf.it \\ ${ }^{4}$ Departamento de Física, Universidad de Atacama, Copayapu 485, Copiapó, Chile
}

A\&A, 605, L4 (2017), https://doi.org/10.1051/0004-6361/201731595

Key words. methods: statistical - techniques: radial velocities - planets and satellites: dynamical evolution and stability errata, addenda

We correct one error that appeared in Zinzi \& Turrini $(2017$, hereafter Paper I).

We report an error in the generation of Fig. 3 in Paper I, showing the preliminary analysis of the anti-correlation between the angular momentum deficit (AMD; Laskar 2000) and multiplicity $(M)$ of exoplanetary systems. The correct plot is shown in Fig. 1 of this corrigendum. As can be seen, albeit a lower value is found for $M=3$ with respect to the trend, the general decreasing trend observed in Paper I is confirmed.

The slope obtained when considering the so-called "strict selection" dataset of Paper I (i.e. data with known uncertainties on the orbital and physical parameters of the exoplanets) appears steeper than the slope associated with that of the "large selection" case (i.e. exoplanets for which the uncertainties are not necessarily available) and seems to fit better the case of TRAPPIST-1.

As discussed in Paper I, this analysis should be only considered preliminary because of the limited number of exosystems in our current dataset, and the AMD-multiplicity anti-correlation will need to be further verified once larger and more precise datasets become available.

Should this anti-correlation be confirmed, as discussed in Paper I, this could indicate that high-multiplicity systems are a common outcome of the planetary formation process, but that a significant portion of these systems form or evolve into unstable orbital configurations.

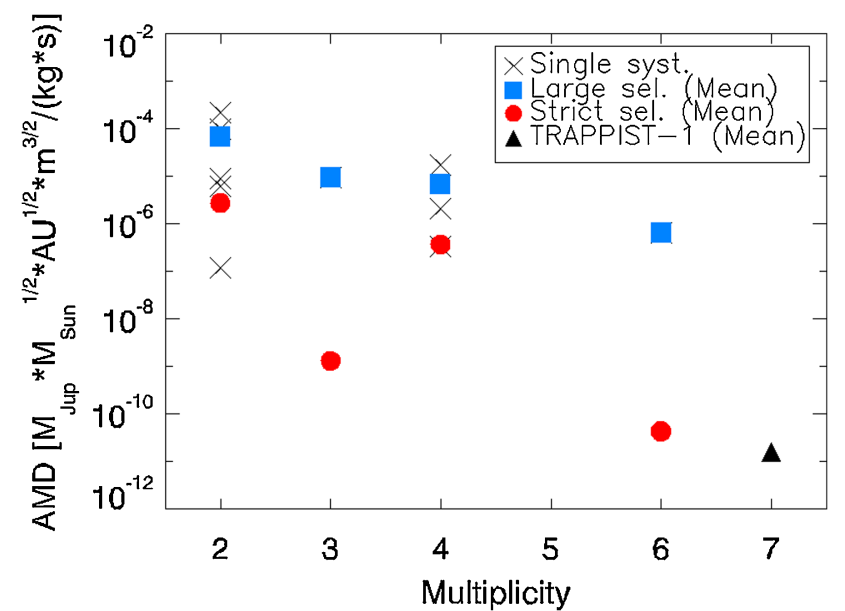

Fig. 1. Angular momentum deficit values of the systems considered. Crosses indicate values for the single systems, blue squares indicate average values for the "large selection" sample, red circles (with error bars smaller than the symbols) indicate averages values for "strict selection" sample. The value of TRAPPIST-1 value is shown as a black triangle.

\section{References}

Laskar, J. 2000, Phys. Rev. Lett., 84, 3240

Zinzi, A., \& Turrini, D. 2017, A\&A, 605, L4 